\title{
Active control of dielectric nanoparticle optical resonance through electrical charging
}

\section{xuebang gao}

Lanzhou University

LiXie ( $\nabla$ xieli@Izu.edu.cn)

Lanzhou University

Jùn Zhou

Lanzhou University

\section{Research Article}

Keywords:

Posted Date: March 2nd, 2022

DOI: https://doi.org/10.21203/rs.3.rs-1372331/v1

License: (c) (i) This work is licensed under a Creative Commons Attribution 4.0 International License. Read Full License 


\section{Abstract}

A novel method to active control of resonance position of dielectric nanoparticles by increasing the excess charge carried by the nanoparticles is proposed in this paper. We show that as the excess charge carried by the particle increases, the oscillation frequency of excess charge will gradually increase, when it is equal to the incident frequency, resonance occurs due to resonant excitation of the excess charge. What is more, the formula of charges carried by an individual particle required to excite the resonance at any wavelength position is proposed. Therefore, the resonance position can be directly controlled by means of particle charging, and the enhancement of resonance intensity is more obvious. This work has opened new avenues for the active control of plasmon resonances, which shows great promise for realizing tunable optical properties of nanoparticles.

\section{Introduction}

Active control of the optical resonance of nanoparticles is of paramount importance to sensing, photocatalysis, photodetection and many other technological applications ${ }^{1,2}$. Active tuning of the resonance positions can be realized by altering the following parameters: the size and shape of the particles $^{3-5}$, the core-to-shell ratio ${ }^{6,7}$, the distance between nanoparticle ${ }^{8}$, material ${ }^{9}$, semiconductor and electronic doping ${ }^{10-13}$, surrounding dielectric material ${ }^{14,15}$, applying electric field ${ }^{16}, 17$, and charge of the nanoparticles ${ }^{18}$. Although the resonance positions of the nanoparticles can be actively tuned by changing the above-mentioned parameters, so far most of such methods have been irreversible ${ }^{1}$. Therefore, significant challenges still remain for active control optical resonance of nanoparticles.

Fortunately, charge induced resonance shifts of extinction spectra due to changes in the free electron density of noble metals nanoparticles have been reported as a reversible process in recent years $3,19,20$. However, Optical resonance can only be blue-shifted by metal nanoparticles plasma charging ${ }^{18}$. But in many actual applications, nanoparticles are often designed for absorption and scattering in specific wavelength regions ${ }^{21,22}$, and currently it is still difficult to flexibly control the resonance position by metal nanoparticles plasma charging. In addition, considering the high cost and rarity of precious metals, the optical resonance of the dielectric nanoparticles caused by charges may have wide application prospects. Actually many dielectric particles are prone to be charged ${ }^{23-25}$, in addition, the atmospheric pressure plasma jet can precisely control how much charges carried by a particle ${ }^{26}$, and charges can be remained up to one week ${ }^{18}$, to make active control of dielectric nanoparticle optical resonance position by charging quite promising. Motivated by this, optical resonances by charged dielectric nanoparticles within the visible and near-infrared wavelength region is investigated in this paper, as shown in Fig. 1, to find a novel method to actively control the resonance position of nanoparticles.

\section{Model Description}


For any charged dielectric nanoparticle with radius $r$ illuminated by light, the calculation of its extinction depends on the electron affinity $\chi$ of the particle, because the existence form of the excess charge carried by the particles is different due to the different electron affinity $\chi^{27}$, as shown in Fig. 2 . For the particles of $\chi<0$, the excess charge carried by the particles is limited to a shell of the atomic scale on the surface of the particles ${ }^{28}$, shown as in Fig. 2(a), while for the micro-nano sized particles of $\chi>0$, the excess electrons will be uniformly distributed in the particles ${ }^{29}$, shown as in Fig. 2(b). The optical properties of charged dielectric nanoparticles can be studied by the core-shell model for particles of $\chi<0$ and by the equivalent model for particles of $\chi<0$.

For the particles of $\chi<0$, the charges will be limited within a shell with thickness $t_{s}\left(t_{s}=2\{\backslash \mathrm{AA}\}^{28}\right)$, so the radius of the particle core is $r_{1}=r-t_{s}$. When the light or near-infrared wave irradiates on a spherical particle with such a core-shell structure, the corresponding size parameters of the whole particle and the core are $y=2 \Pi r / \lambda$ and $x=2 \Pi r_{1} / \lambda$, respectively, in which $\lambda$ is the wavelength and the corresponding angular frequency $\omega=2 \pi c / \lambda$, and $c$ is light speed in vacuum, $3.0 \times 10^{8} \mathrm{~m} / \mathrm{s}$. The permittivity of the shell $\varepsilon_{s}=\varepsilon_{r}+i \sigma_{s} / \omega \varepsilon_{0}$, and the dielectric constant of the corresponding core $\varepsilon_{r}=\varepsilon_{\text {real }}+i \varepsilon_{\text {imag }} \sigma_{s}$ is the bulk conductivity of the shell, $\sigma_{s}=i e^{2} n_{s} / m_{e}(\omega+i \gamma)$, in which $i=\sqrt{-1}$, and $n_{S}$ is the electron density of the shell, $n_{S}=3 q / 4 \Pi\left(r^{3}-r_{1}{ }^{3}\right) e$, where $q$ is the charge of the particle; $e$ is the charge of a single electron, $e=1.6 \times 10^{-19} \mathrm{C} ; m_{e}$ is the mass of the electron, $m_{e}=9.109 \times 10^{-31} \mathrm{~kg} ; \gamma$ is the damping coefficient ${ }^{30}$, and $\gamma=\gamma_{C} k_{B} T / \hbar$, with the Boltzmann constant $k_{B}=1.38 \times 10^{-23} \mathrm{JK}^{-1}$ and the reduced Planck constant $\hbar=1.0546 \times 10^{-34} \mathrm{Js}$ and the temperature $T$. Further the permittivity of the charge shell can be reorganized as $\varepsilon_{s}=\varepsilon_{r}-\omega_{s}^{2} /\left(\omega^{2}+i \omega \gamma\right)$, where $\omega_{s}=\sqrt{n_{s} e^{2} /\left(m_{e} \varepsilon_{0}\right)}$ is the plasma frequency associated with collective oscillation of the excess charge, and $\varepsilon_{0}=8.85 \times 10^{-12}$ is the permittivity of vacuum. With the Drude model of dielectric function ${ }^{31}$, the oscillation frequency of excess charge carried by particles can be expressed as $\omega_{r s}=\omega_{s} / \sqrt{-\operatorname{Re}\left(\varepsilon_{r S}\right)+\varepsilon_{r e a l}} \quad(\gamma \ll \omega)$, in which $\operatorname{Re}\left(\varepsilon_{r S}\right)$ is the real part of the permittivity of the shell $\varepsilon_{r s}$ at resonance. According to ${ }^{32}$, the equivalent dielectric function of such charged particle is

$$
\epsilon_{C S}=\frac{v_{C}}{V_{C}+v_{S}} \epsilon_{r}+\frac{v_{S}}{v_{C}+v_{S}} \epsilon_{S}
$$

1

in which $v_{C}$ is the volume of the core and $v_{S}$ is the volume of the shell.

According to Mie scattering theory ${ }^{33}$, the extinction coefficient of core-shell structure is given by

$$
Q_{e x t}(c)=\frac{2}{y^{2}} \sum_{n=1}^{\infty}(2 n+1) \operatorname{Re}\left(a_{n}{ }^{r}+b_{n}{ }^{r}\right)
$$


Where $a_{n}{ }^{r}$ and $b_{n}{ }^{r}$ are the Mie's scattering coefficients (see supplementary material for details).

For the charged particles of $\chi>0$, the excess electrons will be uniformly distributed in the particles ${ }^{27}$. Therefore, the effective dielectric function of this charged particle can be calculated by the dielectric constant $\varepsilon_{r}$ of particle material and the polarizability of excess charges $a$

$$
\varepsilon_{e f f}=\varepsilon_{r}+\alpha=\left(\varepsilon_{r e a l}+\alpha_{r e a l}\right)+i\left(\varepsilon_{\text {imag }}+\alpha_{\text {imag }}\right)
$$

3

where $\alpha=i \sigma_{b} / \varepsilon_{0} \omega$ and $\sigma_{b}$ is the electric conductivity contributed by the excess charges and it can be calculated by $\sigma_{b}=i e^{2} n_{b} / m_{e}(\omega+i \gamma)$, in which $n_{b}$ denotes the number density of excessive electrons of the particle, $n_{b}=3 q / 4 \pi e r^{3}$. Therefore, the polarizability can be organized as $\alpha=-\omega_{b}^{2} /\left(\omega^{2}+i \omega \gamma\right)=-\frac{\omega_{b}^{2}}{\omega^{2}+\gamma^{2}}+i \frac{\omega_{b}^{2} \gamma}{\omega^{3}+\omega \gamma^{2}}$, where $\omega_{b}=\sqrt{n_{b} e^{2} / m_{e} \varepsilon_{0}}$ is the surface plasma frequency. The oscillation frequency of excess charge carried by the particles is $\omega_{r b}=\omega_{b} / \sqrt{\varepsilon_{\text {real }}+2}$ when $\gamma<\omega$. The refractive index $m=\sqrt{\varepsilon_{e f f}}$ can be obtained through its effective dielectric function. Based on Mie scattering theory, the extinction coefficient of charged particle is given by

$$
Q_{\text {ext }}(c)=\left(2 / x^{2}\right) \sum_{n=1}^{\infty}(2 n+1) \operatorname{Re}\left(a_{n}+b_{n}\right)
$$

4

Where $a_{n}$ and $b_{n}$ are scattering coefficients obtained by Bohren and Huffman ${ }^{33}$.

\section{Results And Discussion}

For nanoscale particles, as the charges carried by the particle increasing, the material properties changed as well, and some of their properties are not the same as ones of the corresponding neutral particle any more, such as the equivalent dielectric function of individual charged $\mathrm{SiO}_{2}$ and $\mathrm{MgO}$ particles shown as in Figs. 2(c)-(d). In Figs. 2(c)-(d), four representative wavelengths in the visible ( $\lambda=536 \mathrm{~nm}, \lambda=780 \mathrm{~nm})$ and infrared bands $(\lambda=1500 \mathrm{~nm}$ and $\lambda=2500 \mathrm{~nm})$ have been selected, and the radius of $\mathrm{SiO}_{2}$ and $\mathrm{MgO}$ particles are 20nm. From Figs. 2(c)-(d), it can be found that with the increase of the charges carried by the particles, the imaginary part of the equivalent dielectric function increases gradually, while the real part of the equivalent dielectric function gradually decreases. The reason is that an increase in charge results in an increase in the density of excess electrons on the particle, and then an increase in excess electrons density affect the equivalent dielectric function of charged nanoparticle, this was also demonstrated by Juluri et al $^{3}$. In addition, the longer the wavelength is, the greater the effect of the 
excess charge on the equivalent dielectric function is. With $\lambda=2500 \mathrm{~nm}$, the real part of the particle's equivalent dielectric function can even reach -154 to see Figs. 2(c)-(d).

Next, for the given incident wave illustrating on a charged particle, the variation of $Q_{\text {ext }}(c) / Q_{\text {ext }}(0)$ (the ratio of the extinction by a charged particle to the one by the corresponding neutral particle), as well as the corresponding imaginary and real parts of the equivalent dielectric function with the charges carried by the particle are shown in Figs. 3(a)-3(c) (MgO particle) and Figs. 3(a1)-3(c1) ( $\mathrm{SiO}_{2}$ particle). From Fig. 3 (b) and Fig. 3(b1), it can be found that the extinction resonance can occur during the particle charging process for both $\mathrm{MgO}$ particle and $\mathrm{SiO}_{2}$ particle although these two particles are with different electron affinity, in which the incident wavelength is $2500 \mathrm{~nm}$. Comparing Fig. 3(b) with Fig. 3(c), for MgO particles with of $r=10 \mathrm{~nm}$ and $r=50 \mathrm{~nm}$, it can be seen that the extinction resonance occurs at $q=8.72 \times 10^{-16} \mathrm{C}$ and $q=1.11 \times 10^{-13} \mathrm{C}$, respectively, and in such situation, it's obvious to find that the real part of the equivalent permittivity of both particles is $\operatorname{Re}\left(\varepsilon_{c s}\right) \approx-4.3$; again comparing Fig. 3(b1) with Fig. 3 (c1), for $\mathrm{SiO}_{2}$ particles of $r=10 \mathrm{~nm}$ and $r=50 \mathrm{~nm}$, extinction resonance occurs at $q=5.1 \times 10^{-16} \mathrm{C}$ and $6.43 \times 10^{-14} \mathrm{C}$, with the corresponding real part of the equivalent permittivity of both these particles $\operatorname{Re}\left(\varepsilon_{\text {eff }}\right) \approx-2$. That means the real part of the equivalent dielectric function can be used to determine if the extinction resonance can be occurred for a given particle when the particle carried excess charges, which also is verified in case of $\lambda=536 \mathrm{~nm}, 780 \mathrm{~nm}, 1550 \mathrm{~nm}$ and $2500 \mathrm{~nm}$ shown as in Fig. 3(d) and Fig. 3(d1) (r= $10 \mathrm{~nm})$.

Further, let's reveal why the extinction resonance occurs during the particle charging process. As excess charge density increases, the oscillation frequency of the excess charge has been changed, as shown in the insert in Fig. 3(e) and Fig. 3(e1). It can be found that the oscillation frequency increases with the increase of excess charges carried by the particle, and extinction resonance occurs if only the oscillation frequencies of the excess charges are equal to the incident wave frequencies to see the Fig. 3(e) and Fig. $3(\mathrm{e} 1)$. This reveals that a charged dielectric nanoparticle whether with positive electron affinity or negative electron affinity, the extinction resonance can occur, because when the oscillation frequency of the excess charge carried by the particle close to the frequency of the incident electromagnetic wave, it will result in resonant excitation.

Further the contours of $\log _{10}\left(Q_{\text {ext }}(C) / Q_{\text {ext }}(0)\right)$ of the charged $\mathrm{MgO}$ particle and the $\mathrm{SiO}_{2}$ particle are displayed with particle size and excess charges at two wavelengths $(\lambda=2500 \mathrm{~nm}$ and $\lambda=1550 \mathrm{~nm})$ shown as in Figs. 4(a1, a2)-4(b1, b2). It can be found that given the wavelength, for different particle sizes of dielectric nanoparticles, the extinction resonance can be occurred if only that the particle can carry suitable charges shown as the bright band in Figs. 4(a1) - (a2) and Figs. 4(b1, b2). Further, for given particle size and selected resonance position, the quantity of charges carried by individual particle to excite extinction resonance is derived as,

$$
q_{\text {rensonance }}=\frac{4 \Pi \epsilon_{0} m_{e}\left(\epsilon_{r e a l}-\operatorname{Re}\left(\epsilon_{r s}\right)\right)}{3 e}\left(r^{3}-r_{1}^{3}\right) \omega^{2}(\chi<0)
$$




$$
q_{\text {rensonance }}=\frac{4 \Pi \epsilon_{0} m_{e}\left(\epsilon_{\text {real }}+2\right)}{3 e} r^{3} \omega^{2}(\chi>0)
$$

6

For the selected resonance position, $q_{\text {rensonance }}$ to excite the resonance directly depends on the particle size shown as in Fig. 4(c). While for given the particle size, the charges to excite resonance at $\lambda=1550 \mathrm{~nm}$ is more than the ones at $\lambda=2500 \mathrm{~nm}$. Theoretically speaking, for any selected resonance position, if the particle size is given, the charges to excite the resonance can be calculated by formula (5) or (6). However, the quantity of charges carried by an individual particle is limited by breakdown voltage which related to the particle material, ambient pressure, etc. ${ }^{34}$ Actually the charges carried by an individual particle cannot be infinite and have a saturation value, e.g. the saturation charges of Martian dust shown as the red line in Fig. 4(c) ${ }^{35}$. Therefore, if the charges required to excite the resonance are beyond the saturation value, the resonance cannot be occurred, while if the charges less than the corresponding saturation value the resonance can be realized such as $\mathrm{MgO}$ particles smaller than $63 \mathrm{~nm}, \mathrm{SiO}_{2}$ particles smaller than $82 \mathrm{~nm}$, the extinction resonance can be occurred when $\lambda=2500 \mathrm{~nm}$. In addition, it can be found from Fig. 4(c) that the resonance charge required to excite the resonance is less for $\mathrm{SiO}_{2}$ particle than for $\mathrm{MgO}$ particle. Therefore, the resonant charge formula given here can be referred to in practical applications to select a more suitable particle material to achieve resonance at less resonant charge.

Finally, the extinction coefficients of the charged $\mathrm{SiO}_{2}$ nanoparticles at resonance position are calculated shown as in Fig. 5, which is also compared with the extinction resonance values of $\mathrm{SiO}_{2} @ A u$ nanoparticles. In Fig. 5, the white circles indicate the extinction coefficients of neutral $\mathrm{SiO}_{2}$ particles with given sizes, and the white core with yellow shell indicate the extinction coefficients of $\mathrm{SiO}_{2} @ \mathrm{Au}$ nanoparticles at the resonance position, and at the same resonance position, the red spheres indicate the extinction coefficients of charged $\mathrm{SiO}_{2}$ particles. Here, parameters such as resonance position, $\mathrm{SiO}_{2} \mathrm{Core}$ radius and $A u$ shell thickness are selected from existed studies $6,9,21,36,37$, as detailed in the supplementary material. From Fig. 5 , it can be found the charges induced resonance is much high than the one by coating $\mathrm{Au}$. That means the charges induced resonance can not only precisely control the resonance position, but also improve the intensity of extinction resonance.

\section{Conclusions}

To conclude, a new method to actively control optical resonance by increasing the quantity of charge carried by the particles is proposed. Our results suggest that extinction resonance can be generated by increasing the particle charges in the visible and infrared bands. And also further explains the reason for the occurrence of resonance, which is caused by the resonant excitation of excess charge carried by the particle. Furthermore, the quantity of charge required for exciting resonance is given, which is a function of the nanoparticle size, wavelength, and dielectrics properties of particles. Finally, dielectric 
nanoparticles can resonate at any wavelength position by particle charging and the generation of larger extinction resonance values. Therefore, this method can be used to realize a height-adjustable optical resonance, and is a new idea for controlling the optical resonance of nanoparticles in technical applications.

\section{Declarations}

FUNDING. This research is supported by grants of the National Natural Science Foundations of China (No.11472122) and the Fundamental Research Funds for the Central Universities (No. Izujbky-2018121).

ACKNOWLEDGMENTS. The authors would like to sincerely thank the reviewers for their valuable comments on this work.

Disclosures. The authors declare no conflicts of interest.

DATA AVAILABILITY. The data that support the findings of this study are available from the corresponding author upon reasonable request.

\section{References}

1. C. P. Byers, H. Zhang, D. F. Swearer, M. Yorulmaz, B. S. Hoener, D. Huang, A. Hoggard, W.-S. Chang, P. Mulvaney and E. Ringe, Science advances 1 (11), e1500988 (2015).

2. H. Xin, B. Namgung and L. P. Lee, Nature Reviews Materials 3 (8), 228-243 (2018).

3. B. K. Juluri, Y. B. Zheng, D. Ahmed, L. Jensen and T. J. Huang, The Journal of Physical Chemistry C 112 (19), $7309-7317$ (2008).

4. E. A. Coronado and G. C. Schatz, The Journal of chemical physics 119 (7), 3926-3934 (2003).

5. H. Wu, X. Cheng, H. Dong, S. Xie and S. He, Nano Research 15 (2), 838-844 (2022).

6. A. Saini, T. Maurer, I. I. Lorenzo, A. R. Santos, J. Béal, J. Goffard, D. Gérard, A. Vial and J. Plain, Plasmonics 10 (4), 791-796 (2015).

7. X. Guo, Z. Hu, J. Lv, H. Li, Q. Zhang, L. Gu, W. Zhou, J. Zhang and S. Hu, Nano Research 15 (2), 12881294 (2022).

8. J.-G. Wang, J. S. Fossey, M. Li, T. Xie and Y.-T. Long, ACS applied materials \& interfaces 8 (12), 83058314 (2016).

9. R. Bardhan, N. K. Grady, T. Ali and N. J. Halas, ACS nano 4 (10), 6169-6179 (2010).

10. I. Kriegel, F. Scotognella and L. Manna, Physics Reports 674, 1-52 (2017).

11. S. Pan, X. Li and J. Yadav, Physical Chemistry Chemical Physics (2021).

12. C. Lu, X. Li, Q. Wu, J. Li, L. Wen, Y. Dai, B. Huang, B. Li and Z. Lou, ACS Nano 15 (2), 3529-3539 (2021).

13. A. Manjavacas and F. J. García de Abajo, Nature Communications 5 (1), 3548 (2014). 
14. M. Salvador, B. A. MacLeod, A. Hess, A. P. Kulkarni, K. Munechika, J. I. Chen and D. S. Ginger, ACS nano 6 (11), 10024-10032 (2012).

15. Y. Chen, X. Ding, S.-C. Steven Lin, S. Yang, P.-H. Huang, N. Nama, Y. Zhao, A. A. Nawaz, F. Guo and W. Wang, ACS nano 7 (4), 3306-3314 (2013).

16. J. Müller, C. Sönnichsen, H. Von Poschinger, G. Von Plessen, T. Klar and J. Feldmann, Applied Physics Letters 81 (1), 171-173 (2002).

17. Y. Gao, X. Li, W. Liu, X. Xing, H. Long, K. Wang, B. Wang and P. Lu, Nano Letters 21 (24), 10230-10237 (2021).

18. M. Ian Lapsley, A. Shahravan, Q. Hao, B. Krishna Juluri, S. Giardinelli, M. Lu, Y. Zhao, I.-K. Chiang, T. Matsoukas and T. Jun Huang, Applied physics letters 100 (10), 101903 (2012).

19. B. Gerislioglu, A. Ahmadivand and N. Pala, Plasmonics 13 (6), 1921-1928 (2018).

20. C. Liow, F. Meng, X. Chen and S. Li, The Journal of Physical Chemistry C 118 (47), 27531-27538 (2014).

21. A. M. Gobin, M. H. Lee, N. J. Halas, W. D. James, R. A. Drezek and J. L. West, Nano Letters 7 (7), $1929-$ 1934 (2007).

22. R. Weissleder, Nature biotechnology 19 (4), 316-317 (2001).

23. V. Fortov, A. Nefedov, V. Molotkov, M. Poustylnik and V. Torchinsky, Physical review letters 87 (20), 205002 (2001).

24. E. W. B. Gill, Nature 162 (4119), 568-569 (1948).

25. J. F. Kok and N. O. Renno, Physical Review Letters 100 (1), 014501 (2008).

26. S. Matsusaka, Advanced Powder Technology 30 (12), 2851-2858 (2019).

27. R. L. Heinisch, F. X. Bronold and H. Fehske, Physical review letters 109 (24), 243903 (2012).

28. D. Geldart and P. Chýlek, Journal of Quantitative Spectroscopy and Radiative Transfer 70 (4-6), 697708 (2001).

29. R. Heinisch, F. Bronold and H. Fehske, Physical Review B 83 (19), 195407 (2011).

30. J. Klačka and M. Kocifaj, Journal of Quantitative Spectroscopy \& Radiative Transfer 106 (1), 170-183 (2007).

31. X. Fan, W. Zheng and D. J. Singh, Light: Science \& Applications 3 (6), e179-e179 (2014).

32. A. Kuzma, M. Weis, S. Flickyngerova, J. Jakabovic, A. Satka, E. Dobrocka, J. Chlpik, J. Cirak, M. Donoval and P. Telek, Journal of Applied Physics 112 (10), 103531 (2012).

33. C. F. Bohren and D. R. Huffman, Absorption and scattering of light by small particles. (1983).

34. M. Di Renzo and J. Urzay, Nature Communications 9 (1), 1676 (2018).

35. A. A. Mills, Nature 268 (5621), 614-614 (1977).

36. L. R. Hirsch, J. B. Jackson, A. Lee, N. J. Halas and J. L. West, Analytical Chemistry 75 (10), $2377-2381$ (2003).

37. Y. Lu, J. Zhong, G. Yao and Q. Huang, Sensors and Actuators B: Chemical 258, 365-372 (2018). 


\section{Figures}

\section{Figure 1}

Charge-induced extinction resonance of dielectric nanoparticles

\section{Figure 2}

A schematic diagram of light irradiating on charged particles with (a) positive and (b) negative electron affinity, real and imaginary parts of the charges-dependent function of (c) $\mathrm{MgO}$ and (d) $\mathrm{SiO}_{2}$, the radius of the charged particle is $r=20 \mathrm{~nm}$.

\section{Figure 3}

For $\mathrm{MgO}$ and $\mathrm{SiO}_{2}$ particles with radius of $r=10 \mathrm{~nm}$ and $r=50 \mathrm{~nm}$, with the increase of the charge carried by the particles, the changes of the imaginary part of the equivalent dielectric function of $\mathrm{MgO}$ and $\mathrm{SiO}_{2}$ particles $(\mathrm{a}, \mathrm{a} 1)$ and the real part (c, $\left.\mathrm{c} 1\right)$ change, the wavelength is $2500 \mathrm{~nm}$. The variation of $Q_{e x t}(c) / Q_{e x t}(0)$ with the quantity of charge carried by particles $(\mathrm{b}, \mathrm{b} 1)$ and the ratio of resonance frequency of excess charge to incident frequency (e, e1). $Q_{\text {ext }}(c) / Q_{\text {ext }}(0)$ of (d) MgO particle and (d1) $\mathrm{SiO}_{2}$ particle varying with the real part of the equivalent dielectric in case of $\lambda=536 \mathrm{~nm}, 780 \mathrm{~nm}, 1550 \mathrm{~nm}$ and $2500 \mathrm{~nm}$, and $r=10 \mathrm{~nm}$.

\section{Figure 4}

Contour of $\log _{10}\left(Q_{e x t}(c) / Q_{e x t}(0)\right)$ varying with the particle size and the excess charges, in which wavelengths are $\lambda=2500 \mathrm{~nm}$ and $\lambda=1550 \mathrm{~nm}$, (a1, a2) $\mathrm{MgO}$ particle, (b1, b2) $\mathrm{SiO}_{2}$ particle. (c) indicate the quantity of charge required for resonance to occur with varying particle size at different wavelengths, and the red solid lines in (c) mean that charges of the particles with given size reach maximum.

\section{Figure 5}

The extinction coefficient of neutral $\mathrm{SiO}_{2}$ nanoparticles (white spheres), $\mathrm{SiO}_{2} @ A$ u core shell nanoparticles (white spheres core yellow shell) after $\mathrm{SiO}_{2}$ nanoparticles wrapping Au shell, charged $\mathrm{SiO}_{2}$ nanoparticles 
(red spheres) after $\mathrm{SiO}_{2}$ nanoparticles charging at the resonance positions of $\mathrm{SiO}_{2} @ \mathrm{Au}$ core shell nanoparticles, the numbers above the red sphere and below the core-shell sphere indicate the magnification of extinction amplification by charging and by wrapping the Au shell, respectively.

\section{Supplementary Files}

This is a list of supplementary files associated with this preprint. Click to download.

- SupplementaryMaterials22.pdf 